\title{
Frequency-stabilised laser reference system for water vapour spectroscopy and sensing applications
}

\author{
R. Matthey, C. Affolderbach, G. Mileti \\ Observatoire de Neuchâtel, Observatoire 58, CH-2000 Neuchâtel, Switzerland, renaud.matthey@ne.ch \\ S. Schilt, D. Werner, L. Thévenaz \\ Ecole Polytechnique Fédérale de Lausanne (EPFL), Laboratory of Nanophotonics and Metrology, \\ CH-1015 Lausanne, Switzerland, stephane.schilt@epfl.ch
}

Water vapour is a major atmospheric constituent in terms of impact on climate and weather. Since measurement data on its highly variable distribution in time and space are crucial but not available to a satisfactory extend, the European Space Agency (ESA) has been considering a satellite-borne differential-absorption lidar (DIAL) instrument in the frame of the "Water Vapour Lidar Experiment in Space" (WALES) mission. The DIAL technique offers the advantages of high vertical resolution and selective gas detection by sensing the difference in light absorption at two close wavelengths, one of which coinciding with an absorption line and the second one usually chosen out of any line. For reliable water vapour lidar measurements, high spectral purity, short- and long-term stabilities and high optical power of the transmitter laser are necessary. A way to achieve these requirements is to inject the cw output of a low-power frequency detection unit (FDU) that fulfils the necessary stability and spectral purity conditions into a pulsed power oscillator.

We conceived and are realising such a FDU for water vapour spectroscopy. Even if it is mainly a demonstrator of principle, constraints for space application (weight, size, power consumption, redundancy) were considered though not pushed to their full possible limits. The large range of water vapour concentration in the atmosphere is addressed by three different wavelengths centred on three water vapour lines of different absorption cross-sections. The on-line wavelengths share a common off-line wavelength; all lines lie within $0.3 \mathrm{~nm}$ around $935.4 \mathrm{~nm}$. The approach to dedicate a separate injection seed laser (ISL) for each wavelength was chosen for frequency stability, design simplicity, system reliability and redundancy. The ISL's have to display narrow linewidth (less than $10 \mathrm{MHz}$ ), good passive stability (freerunning frequency drift less than $500 \mathrm{MHz}$ on a 24-hour basis) and high spectral purity. Due to their small size, low power consumption, reliability and frequency-tuning capability, semiconductors lasers suit best the application. Two types of lasers are currently under test, distributed-feedback (DFB) lasers and extended-cavity diode lasers (ECDL's). DFB lasers show large mode-hop-free tuning range and, as single-chip laser without any mechanical part, can potentially demonstrate very high reliability. However, they are scarcely available at $935 \mathrm{~nm}$ and have a limited total tuning range ( $5 \mathrm{~nm}$ typically). We have developed and realised an ECDL (320-cm3 volume), based on our previous design of a compact and highly stabilised ECDL laser head, using home-made laser control and laser locking electronics [1]. Preliminary tests and results including heterodyne measurements on the individual ISL's anticipate performances within the target specifications. Due to its large tuning range, the ECDL can be adjusted at $935 \mathrm{~nm}$ or at $942 \mathrm{~nm}$, a region also considered as possible for the WALES transmitter.

The second main subsystem of the FDU is a frequency reference unit (FRU). Its task is to grant short- and longterm frequency stabilities of the ISL's ( $<50 \mathrm{MHz}$ at $20 \mathrm{~s}$, and $<60 \mathrm{MHz}$ over 2 years). It includes a water vapour reference and four frequency stabilisation mechanisms to lock the ISL's on and off the water vapour lines. For reasons of optical adjustment and redundancy, three different compact absorption cells filled with low-pressure water vapour are used, one per on-line laser. The selected design implements multipass configurations with different cell path lengths (from $75 \mathrm{~cm}$ up to $4 \mathrm{~m}$ ) in order to improve the absorption signals. As the absorption line strengths are very different (more than two orders of magnitude) and as one wavelength has to be set in a spectral region free of absorption, the frequency stabilisation scheme has been designed in a differentiated approach. Stabilisation of the three on-line ISL's is performed by wavelength modulation spectroscopy (WMS) with first harmonic detection. A theoretical model, including the effect of both laser frequency- and intensity-modulation, has been developed to simulate the harmonic signals to be used for laser locking [2]. This theoretical analysis has enabled to determine a suitable modulation/demodulation scheme for each of the ISL's. For the weakest absorption line, a balanced detection is used to remove the $1 f$-background level produced by the laser intensity modulation. The off-line locking is realised with an offset-locking between the off-line laser and one on-line laser. For this purpose, an electrical filter is used to simulate an artificial microwave absorption line and acts as a frequency discriminator to lock the beat note between the two lasers at a fixed and precisely selected value. The designed method is very similar to the WMS technique used for the direct laser stabilisation on the water lines, but it applies to the beat note in the electrical domain. Offset-locking with frequency differences up to $19 \mathrm{GHz}$ has been experimentally demonstrated with the proposed technique.

Results on the frequency stability and spectral properties of the system will be presented and discussed.

[1] C. Affolderbach, G. Mileti, Optics and Lasers in Engineering 43 (3-5), 291-302 (2005).

[2] S. Schilt, L. Thévenaz, P. Robert, Appl. Opt. 42 (33), 6728-6738 (2003) 